\title{
Validación de instrumento para identificar el nivel de vulnerabilidad de los trabajadores de la salud a la tuberculosis en instituciones de salud (IVTS TB-001)
}

\author{
Validation of instrument to identify the vulnerability level of health workers to \\ tuberculosis in health institutions (IVTS TB-001)
}

\section{Óscar Andrés Cruz Martínez', Alba Idaly Muñoz Sánchez²}

1. Universidad Nacional de Colombia. Magister en Salud y Seguridad en el Trabajo. Universidad Nacional de Colombia.

2. Universidad del Valle. Universidad de Sao Paulo Brasil.

\section{Recibido: 03-11-15}

Aceptado: 19-12-15

\section{Correspondencia}

Alba ldaly Muñoz Sánchez

Enfermera. Universidad del Valle. Especialista en Salud Ocupacional, Magister y Doctora en

Enfermería con Énfasis en Salud Colectiva. Universidad de Sao Paulo Brasil.

Docente Titular Facultad de Enfermería- Universidad Nacional de Colombia.

Teléfono: I 3165000 ext 17082.

Correo electrónico: aimunozs@unal.edu.cos

\section{Resumen}

La Salud y Seguridad en el trabajo son una prioridad en los trabajadores que cuidan la salud, dada su permanente exposición a factores de riesgo en el proceso de trabajo que desarrollan. De forma particular, la exposición a la tuberculosis en los trabajadores sanitarios, se considera una problemática frecuente y relevante en su estudio, lo cual precisa la necesidad de desarrollar procesos metodológicos y de validación de instrumentos sólidos, que contribuyan a la identificación e intervención de los factores de vulnerabilidad de los trabajadores del sector salud a la tuberculosis.

Objetivo: Diseñar y validar un instrumento que permita identificar el nivel de vulnerabilidad de los Trabajadores de la Salud a la Tuberculosis en Instituciones de Salud.

Materiales y Métodos: Estudio de tipo metodológico, correspondiente al diseño y validación de un instrumento en sus fases de validación aparente y validación de contenido, que permita identificar el nivel de vulnerabilidad de los Trabajadores de la Salud a la Tuberculosis en Instituciones de Salud

Resultados: Se efectuó el diseño del instrumento, en sus 4 dimensiones, 19 subdimensiones y 50 ítems, con su posterior validación aparente en la población objeto, mediante la valoración de la comprensibilidad, en una muestra aleatoria de $\mathrm{N}^{\circ}=(502)$ trabajadores de la salud de dos instituciones en las cuales se brinda atención a pacientes con diagnóstico de tuberculosis. La valoración de la Comprensibilidad del instrumento en los trabajadores, fue del (97,8\%). De igual manera, se realizó juicio de expertos y se obtuvo un índice mayor de $>0.58$ en la comprensión de los ítems con $\mathrm{N}=(0.90)$, una precisión del $\mathrm{N}=(0.90)$, y una claridad del $\mathrm{N}=(0.92)$. En la validación de contenido se obtuvo una razón de validez de Lawshe modificado por Tristán del $\mathrm{N}=(0,91)$, demostrando características de validez aparente y de contenido.

Conclusión: El diseño y validación del instrumento (IVTS TB 001), a nivel de validez aparente obtuvo comprensión de los ítems en la población objeto de estudio. Así mismo, obtiene resultados de comprensión, precisión, claridad, y en la validez de contenido en la relevancia de los ítems dada por los expertos, lo cual 
lo convierte en una herramienta de gran utilidad en el campo de la Salud y Seguridad en el Trabajo en el sector salud, y en la evaluación de los factores de vulnerabilidad de los trabajadores de la salud a la tuberculosis.

Med Segur Trab (Internet) 2015; 61 (241) 448-467

Palabras Claves DeCS: ocupacional, tuberculosis, trabajadores de la salud, validación de instrumentos, vulnerabilidad en salud.

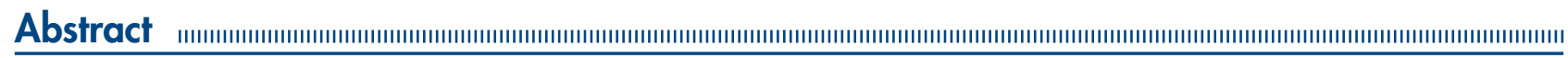

Health and Safety at work is a priority for health care workers, given their permanent exposure to hazardous agents in the process of work. In particular, exposure to TB in health care workers is considered a common and important problem to be studied, which, in turn, requires the need to develop methodological processes and validation of robust tools that contribute to the identification and intervention vulnerabilities of health workers in the tuberculosis sector.

Objective: To design and validate a tool to identify the level of vulnerability of Health Workers TB in health facilities.

Materials and Methods: methodological, design and validation of an instrument validation phases apparent and content validation, to identify the vulnerability level of Health Workers TB in health institutions.

Results: made the design of the instrument, in its 4 dimensions, 19 subdomains and 50 items, with subsequent validation apparent in the target population, by assessing the comprehensibility, in a random sample of No. $=(502)$ health workers, two institutions in which care is offered to patients diagnosed with tuberculosis. The assessment of the instrument comprehensibility in the target population was (97.8\%). Similarly, in the judgment of experts a higher rate of $>0.58$ in understanding the items with $\mathrm{N}=(0.90)$, accuracy $N=(0.90)$, and clarity of the $N=(0.92)$ was obtained. In the content validation a reasonable Lawshe validity, amended by Tristan $N=(0.91)$, was met, demonstrating the characteristics of face and content validity instrument.

Conclusion: The design and validation of the instrument (IVTS TB-001) at the level of validation apparent understanding of the items obtained in the study population. Also, results obtained in assessing face validity of understanding, accuracy, clarity, and content validity in the relevance of the items given by experts, which makes it a very useful tool in the field of Health and Safety at Work in the health sector, which enables the identification of the level of health vulnerability workers in regards to tuberculosis in health institutions and the evaluation of the vulnerabilities of Health Workers to tuberculosis.

Med Segur Trab (Internet) 2015; 61 (241) 448-467

Keywords (BVS-DeCs): Occupational Tuberculosis, Health Workers, validation of instruments, bealth vulnerability. 


\section{INTRODUCCIÓN}

La estrecha relación de la Salud y el Trabajo como categorías permeadas por aspectos histórico-sociales, sociopolíticos, económicos entre otros, ameritan un estudio profundo de la Salud y Seguridad en los diferentes escenarios de trabajo, espacios donde los individuos y colectivos de trabajadores desarrollan sus potencialidades, pero donde también, pueden estar expuestos a diferentes agentes de riesgo que aumentan su vulnerabilidad de enfermar generando efectos adversos y/o daños en su salud. ${ }^{1}$

En tanto, se reconoce necesario observar como la influencia del trabajo en la salud de los trabajadores, se ve afectada por patrones específicos de desgaste, y procesos alternados de Salud- Enfermedad. La determinación social de la salud y enfermedad en los trabajadores, y los fenómenos de la flexibilización y precarización laboral en el mundo actual del trabajo, impactan de manera negativa en las condiciones de trabajo, el estado de salud, y las dimensiones individual/extralaboral, de los trabajadores del sector. $^{2}$

La Organización Internacional del Trabajo (OIT), ha priorizado los trabajadores sanitarios, como población en la que se debe asegurar condiciones favorables en torno a su Salud y Seguridad en el Trabajo dada, su exposición permanente a condiciones de trabajo adversas, derivadas de la exposición a contaminantes químicos, físicos biológicos, alta carga mental y física, así como también, la influencia de formas atípicas de vinculación laboral generando afectación de su salud y que a su vez afecta la calidad en la atención en salud. ${ }^{3}$

De igual manera podemos mencionar que las Condiciones y Medio Ambiente de Trabajo (CYMAT), particularmente en el sector salud, existen agentes de riesgos definidos como el conjunto de propiedades, y características de una situación de trabajo de manera particular y que influyen de manera preponderante en la salud de los trabajadores; estas son el resultado de factores de riesgo socio técnicos, organizacionales y del medio ambiente de trabajo. ${ }^{4}$

Por su parte, la tuberculosis es considerada una grave problemática en la salud pública en el mundo y en Colombia, siendo la segunda causa de muertes derivadas de enfermedades de tipo infectocontagiosas en la población general, verificado por sus indicadores epidemiológicos en su persistencia. ${ }^{5}$ Esta enfermedad, trasciende el campo de interés de la salud pública, a la salud laboral, en razón de que la exposición de los trabajadores del sector salud en sus escenarios de trabajo, puede ocasionar contagios con sepas sensibles o resistentes en los trabajadores siendo así considerada una enfermedad prioritaria y de origen laboral en varios países del mundo. ${ }^{6}$

Según últimos datos reportados por la Organización Mundial de la Salud (OMS), en el mundo, se diagnosticaron para el año 2014 un total de 9 millones de casos nuevos de tuberculosis, de los cuales se registraron 1 millón de muertes y hubo cerca de 1,2 millones de casos asociados a la coinfección TB/VIH. En relación a los casos de Tuberculosis en los trabajadores de la salud, algunas cifras estiman un riesgo de contagio de 10 a 100 veces mayor en relación a la población en general. ${ }^{7}$ En algunos países de mayor carga de la enfermedad se estiman tasas que varían entre los 2 a los 2038 casos por cien mil trabajadores con diagnóstico de tuberculosis; ${ }^{5}$ Particularmente en Colombia se reportaron en el 2014, una cifra cerca de 150 casos de Tuberculosis en Trabajadores de la Salud, y en la ciudad de Bogotá 36 casos, lo cual indica una problemática frecuente de relevante interés en el ámbito de la salud laboral de los trabajadores del sector salud. ${ }^{8}$

La exposición a la Tuberculosis en los Trabajadores de la Salud puede ser considerada una problemática compleja y poco estudiada en Latinoamérica y en Colombia, en la cual se hace necesario desarrollar procesos investigativos y de validación de instrumentos, que brinden soporte a estudios diagnósticos y de intervención. Por lo tanto, el presente artículo de investigación mostrará los resultados del proceso de diseño y validación de un 
instrumento para identificar el nivel de vulnerabilidad de los trabajadores de la salud a la tuberculosis en instituciones de salud en sus siglas IVTS TB -001. Este instrumento integra en su enfoque conceptual, las dimensiones individual/extralaborales, las condiciones de trabajo, las condiciones de salud y el control de la infección, siendo categorías de gran relevancia en el análisis de la problemática objeto de estudio.

De igual manera, para el desarrollo del instrumento IVTS TB- 001, se tomó como constructo conceptual la Vulnerabilidad en salud, definida como; «la susceptibilidad o los factores protectores de los trabajadores de la salud a problemas y/o daños en su salud a nivel individual y colectivo". Particularmente en torno a este constructo diferentes autores, evidencian que pueden existir diferentes niveles de afectación o vulnerabilidad de enfermar por tuberculosis, marcados por la influencia de las condiciones de vida, de trabajo y de salud. ${ }^{9}$

\section{OBJETIVO}

Diseñar y validar un instrumento que permita identificar el nivel de vulnerabilidad de los Trabajadores de la Salud a la Tuberculosis en Instituciones de Salud.

\section{MATERIALES Y MÉTODOS}

El desarrollo del proceso metodológico de esta investigación correspondió a un estudio de tipo metodológico, en el cual se diseñó y validó un instrumento a nivel de validez aparente y de contenido, que permitiera identificar el nivel de vulnerabilidad de los Trabajadores de la Salud a la Tuberculosis en Instituciones de Salud. Por su parte, los estudios metodológicos, son aquellas investigaciones indispensables para la construcción del conocimiento, en cualquier campo de estudio, en especial de aquellos fenómenos complejos relacionados a la salud, y que integran diferentes categorías asociadas en su análisis y base fundamental en la construcción de instrumentos. ${ }^{10}$

La validez se define como la propiedad de que un instrumento mida lo que realmente pretende medir. ${ }^{11}$

La validez aparente, también denominada "validez de presentación", o "validez facial", se refiere a la apreciación subjetiva que se produce en las personas (sujeto de estudio o expertos), ante la revisión general del instrumento, sin entrar en detalles de diseño o de calidad del enfoque o del contenido. ${ }^{12}$

La validez de contenido, se relaciona al grado en que el instrumento a ser utilizado reflejan el dominio específico de las dimensiones, subdimensiones y los ítems a ser aplicados. Este tipo de validación, tiene por objeto evitar conclusiones inválidas a partir del cumplimiento de criterios conceptuales, y el alcance de los mismos para explicar el fenómeno objeto de estudio. ${ }^{13}$

Para efectos del proceso de investigación del instrumento para identificar el nivel de vulnerabilidad de los Trabajadores de la Salud a la Tuberculosis en Instituciones de Salud o IVTS TB-001, se desarrollaron las siguientes fases;

Fase I: Aproximación al constructo teórico-conceptual y formulación de las dimensiones, subdimensiones e ítems

La fase número uno correspondió a los procedimientos relacionados a la aproximación para la identificación de los antecedentes teórico-conceptuales del tema objeto de estudio, con el objeto de definir la pertinencia y coherencia en cuanto a la formulación de un instrumento de medición y la problematización del objeto de estudio, así como también la definición del constructo teórico y sus categorías, dimensiones e ítems en torno a la (vulnerabilidad de los trabajadores de la salud a la tuberculosis). 


\section{Fase II: Evaluación inicial del instrumento (Validez facial o aparente)}

Posterior al diseño preliminar del instrumento en el cual se estructuraron las categorías, dimensiones, subdimensiones y los ítems, así como la escala de valoración que conformó el instrumento, se procedió al desarrollo de la validación facial o aparente. El desarrollo del proceso de validación del instrumento para identificar el nivel de vulnerabilidad de los Trabajadores de la Salud a la Tuberculosis en Instituciones de Salud contó con las siguientes etapas subsecuentes:

\section{a) Valoración de la comprensión Lingüística Cuantitativa}

En la etapa de validación aparente a nivel cuantitativa se realizó la valoración de la comprensión lingüística del instrumento en la población objeto; este fue definido mediante criterios estadísticos para poblaciones infinitas a una muestra aleatoria simple de $\mathrm{N}=502$ trabajadores de la salud, de dos instituciones tanto de áreas asistenciales como administrativas, donde se brindaba atención a casos de Tuberculosis:

Tabla 1. Cálculo de muestra estimada de proporciones infinitas validación del instrumento (IVTS TB- 001)

\begin{tabular}{ccc}
\hline Índice de confianza & $97,50 \%$ & \\
\hline $\mathrm{Z}=$ & 0,025 & \\
\hline Error aleatorio estimado $\varepsilon=$ & 0,05 & \\
\hline \multicolumn{4}{c}{$\begin{array}{c}\text { Distribución del Percentil } \\
\text { Estándar: } 2,241402728\end{array}$} \\
\hline Institución & $\mathrm{N}^{\circ}$ trabajadores & Tipo de vinculación \\
\hline Hospital A 49,4\% & 124 & Planta \\
\hline Hospital B 50,6\% & 127 & Contrato \\
\hline Total Selección Muestra & 124 & Planta \\
\hline & 127 & Contrato \\
\hline
\end{tabular}

Luego de la aplicación del instrumento, se sometieron los ítems a revisión por parte de los trabajadores seleccionados, conservando la redacción propuesta si el 95\% o más de los sujetos seleccionados manifestaban que lo comprendían totalmente. El ítem fue objeto de revisión y cambios en verbos, signos de puntuación si el $90 \%$ al 94,9\% de los sujetos reportaban que comprendía parcialmente el ítem, y el ítem sufrió restructuración o remplazo si el porcentaje de sujetos que afirman no comprender el ítem, era igual o inferior al $89,9 \%$.

\section{b) Valoración de la claridad, precisión y comprensión cualitativa.}

En este proceso se efectuó valoración por expertos en torno a la apreciación subjetiva que se producía, ante la revisión general del instrumento y sus ítems, en los siguientes aspectos:

a. Comprensión: Entendimiento de lo que se quiere decir al leer o interpretar el ítem.

b. Claridad: Frase o ítem con lenguaje y redacción que se puede comprender.

c. Precisión: Frase o ítem puntual y exacto a lo que se quiere indicar, y el cual no da lugar a dudas.

Los cálculos de la muestra para poblaciones infinitas se efectuaron con el procesador de Excel 2010 y por asesor estadístico a fin de definir el número de muestra de trabajadores que participaron en el proceso de validación. 


\section{Fase III: Evaluación de la validez de contenido}

La validación de contenido, buscó determinar el grado de correlación de las preguntas incluidas en la escala, y si estas eran equivalente al constructo a ser medido. Para la validación de contenido se utilizaron criterios cualitativos y cuantitativos. ${ }^{14}$

Dentro de los criterios cualitativos se efectúo la validación mediante un juicio de 5 expertos. Según plantea Lawshe, en el juicio de expertos, se puede evaluar criterios de relevancia es decir, si los ítems son relevantes, poco relevantes y no relevantes y llegar a un conceso mediante la aplicación de la fórmula CVT en sus siglas en inglés (Content Validity Ratio) o Tasa de Validación de Contenido ${ }^{15}$. Esta fue modificada por Tristán, en la cual es considerada una valoración aceptable del ítem, cuando se reporta un consenso igual o mayor a la constante $>\mathbf{0 . 5 8 2 3}$, independiente del número de participantes que se incluyan en el juicio de expertos. ${ }^{16}$

\section{RESULTADOS}

Los resultados de diseño y conformación de dimensiones, subdimensiones e ítems del instrumento (IVTS TB- 001), identificaran las categorías asociadas al tema de exposición a la tuberculosis en trabajadores de la salud, para lo cual se estructuraron las dimensiones, subdimensiones, e ítems iniciales.

Así mismo, se constató en la revisión conceptual de Condiciones individuales/ extralaborales, condiciones de Salud, condiciones de trabajo, y de control de la infección tuberculosa, categorías sobre las cuales se integra el constructo de vulnerabilidad de los trabajadores de la salud a la tuberculosis en instituciones de salud, tal como se muestra a continuación:

Tabla 2. Conformación de Categorías, Dimensiones y Subdimensiones e ítems Instrumento (IVTS TB- 001)

\begin{tabular}{|c|c|c|c|}
\hline $\begin{array}{l}\text { Categorías } \\
\text { asociadas }\end{array}$ & Dimensiones & Subdimensiones & Ítems \\
\hline \multirow[t]{9}{*}{$\begin{array}{l}\text { Condiciones } \\
\text { de vida }\end{array}$} & \multirow[t]{9}{*}{$\begin{array}{l}\text { 1. Individual/ } \\
\text { Extralaboral }\end{array}$} & 1. Alimentación & $\begin{array}{l}\text { Compra de alimentos como carnes, leche, } \\
\text { huevos. }\end{array}$ \\
\hline & & $\begin{array}{l}\text { 2. Vivienda y } \\
\text { acceso a servicios } \\
\text { básicos }\end{array}$ & $\begin{array}{l}\text { Necesidades de vivienda como el pago del } \\
\text { arriendo, y el pago de servicios públicos. }\end{array}$ \\
\hline & & $\begin{array}{l}\text { 3. Acceso a servicios } \\
\text { de salud }\end{array}$ & $\begin{array}{l}\text { Pago de servicio de salud y/o compra de } \\
\text { medicamentos. }\end{array}$ \\
\hline & & 4. Educación & $\begin{array}{l}\text { Gastos educativos para realizar cursos, } \\
\text { diplomados, estudios de pregrado o } \\
\text { posgrado. }\end{array}$ \\
\hline & & $\begin{array}{l}\text { 5. Acceso a } \\
\text { información }\end{array}$ & $\begin{array}{l}\text { Servicios de televisión, radio, prensa y/o } \\
\text { internet. }\end{array}$ \\
\hline & & $\begin{array}{l}\text { 6. Actividades } \\
\text { recreativas }\end{array}$ & $\begin{array}{l}\text { Realizar actividades al aire libre como salir a } \\
\text { caminar, viajar, salir al parque u otros. }\end{array}$ \\
\hline & & 7. Capacitación & $\begin{array}{l}\text { Haber recibido capacitaciones sobre } \\
\text { tuberculosis en su formación técnica o } \\
\text { profesional. }\end{array}$ \\
\hline & & \multirow[t]{2}{*}{ 8. Conocimientos } & $\begin{array}{l}\text { Que la tuberculosis continúe transmitiéndose } \\
\text { con el tratamiento. }\end{array}$ \\
\hline & & & $\begin{array}{l}\text { Que la tuberculosis se transmita al compartir } \\
\text { alimentos, dar la mano, o sea hereditaria. }\end{array}$ \\
\hline
\end{tabular}




\begin{tabular}{|c|c|c|c|}
\hline $\begin{array}{l}\text { Categorías } \\
\text { asociadas }\end{array}$ & Dimensiones & Subdimensiones & Ítems \\
\hline \multirow[t]{14}{*}{ Salud } & \multirow{14}{*}{$\begin{array}{l}\text { 2. Condiciones de } \\
\text { salud }\end{array}$} & \multirow{4}{*}{$\begin{array}{l}\text { 9. Hábitos de salud/ } \\
\text { enfermedad }\end{array}$} & Fumar cigarrillo. \\
\hline & & & Tomar alcohol. \\
\hline & & & Consumir drogas o psicoactivos. \\
\hline & & & $\begin{array}{l}\text { Tener conductas sexuales de riesgo sin } \\
\text { protección. }\end{array}$ \\
\hline & & \multirow[t]{6}{*}{$\begin{array}{l}\text { 10. Antecedentes } \\
\text { de salud }\end{array}$} & $\begin{array}{l}\text { Contacto con un familiar y/o amigo que } \\
\text { padeció tuberculosis. }\end{array}$ \\
\hline & & & $\begin{array}{l}\text { Haber sido diagnosticado con cáncer y } \\
\text { recibir tratamiento. }\end{array}$ \\
\hline & & & Diabetes o alteraciones del azúcar. \\
\hline & & & Insuficiencia renal o trasplantes. \\
\hline & & & Tratamiento preventivo para tuberculosis. \\
\hline & & & $\begin{array}{l}\text { Artritis y/o estar bajo tratamiento con } \\
\text { medicamentos como prednisona, cortisona } \\
\text { u otros. }\end{array}$ \\
\hline & & \multirow{4}{*}{$\begin{array}{l}\text { 11. Estado de } \\
\text { salud/síntomas } \\
\text { asociados }\end{array}$} & $\begin{array}{l}\text { Tos y/o expectoración (flemas) de más de } 15 \\
\text { días. }\end{array}$ \\
\hline & & & Cansancio, falta de energía física o fatiga. \\
\hline & & & Fiebre y/o sudoración nocturna. \\
\hline & & & Pérdida de peso. \\
\hline \multirow[t]{13}{*}{ Trabajo } & \multirow[t]{13}{*}{$\begin{array}{l}\text { 3. Condiciones de } \\
\text { trabajo }\end{array}$} & \multirow[t]{2}{*}{$\begin{array}{l}\text { 12. Contaminantes } \\
\text { físicos }\end{array}$} & $\begin{array}{l}\text { Trabajar en lugares oscuros, fríos, y/o } \\
\text { húmedos. }\end{array}$ \\
\hline & & & Equipos de rayos $\mathrm{x}$, o de medicina nuclear. \\
\hline & & \multirow{2}{*}{$\begin{array}{l}\text { 13. Contaminantes } \\
\text { químicos }\end{array}$} & Gases anestésicos, gasolinas, alcoholes. \\
\hline & & & $\begin{array}{l}\text { Medicamentos inmunosupresores como } \\
\text { metrotexato, ciclosfamida u otros. }\end{array}$ \\
\hline & & \multirow[t]{5}{*}{$\begin{array}{l}\text { 14. Contaminantes } \\
\text { biológicos }\end{array}$} & $\begin{array}{l}\text { Atención a pacientes con tuberculosis } \\
\text { pulmonar. }\end{array}$ \\
\hline & & & $\begin{array}{l}\text { Atención a pacientes con diagnóstico de } \\
\mathrm{VIH} / \text { sida. }\end{array}$ \\
\hline & & & $\begin{array}{l}\text { Atención a pacientes con diagnóstico de } \\
\text { tuberculosis resistente a medicamentos. }\end{array}$ \\
\hline & & & $\begin{array}{l}\text { Nebulizaciones, cirugías en el tórax, } \\
\text { intubaciones, y/o desinfección de equipos } \\
\text { respiratorios. }\end{array}$ \\
\hline & & & $\begin{array}{l}\text { Muestras de esputo o flemas, o de secreciones } \\
\text { bronquiales, y/o cultivos de Mycobacterias. }\end{array}$ \\
\hline & & $\begin{array}{l}\text { 15. Condiciones de } \\
\text { la tarea }\end{array}$ & $\begin{array}{l}\text { Un puesto de trabajo en hacinamiento. } \\
\text { Un alto nivel de estrés. }\end{array}$ \\
\hline & & \multirow{3}{*}{$\begin{array}{l}\text { 16. Condiciones de la } \\
\text { organización }\end{array}$} & Turnos nocturnos. \\
\hline & & & Jornadas de más de 12 horas al día. \\
\hline & & & Varias instituciones de salud. \\
\hline
\end{tabular}




\begin{tabular}{|c|c|c|c|}
\hline $\begin{array}{l}\text { Categorías } \\
\text { asociadas }\end{array}$ & Dimensiones & Subdimensiones & Ítems \\
\hline \multirow[t]{13}{*}{$\begin{array}{l}\text { Trabajo } \\
\text { (cont.) }\end{array}$} & \multirow{13}{*}{$\begin{array}{l}\text { 4. Control de } \\
\text { la infección } \\
\text { tuberculosa }\end{array}$} & \multirow{5}{*}{$\begin{array}{l}\text { 17. Medidas } \\
\text { administrativas/ } \\
\text { programático }\end{array}$} & $\begin{array}{l}\text { Demora en el diagnóstico y/o tratamiento de } \\
\text { pacientes con tuberculosis pulmonar. }\end{array}$ \\
\hline & & & Pacientes con tos y/o expectoración. \\
\hline & & & $\begin{array}{l}\text { Cumplimiento de un protocolo de } \\
\text { bioseguridad para la atención de casos de } \\
\text { tuberculosis. }\end{array}$ \\
\hline & & & $\begin{array}{l}\text { Material educativo para prevenir casos de } \\
\text { tuberculosis en el personal de salud. }\end{array}$ \\
\hline & & & $\begin{array}{l}\text { Le han aplicado la prueba de tuberculina } \\
\text { para identificar infección latente por } \\
\text { tuberculosis. }\end{array}$ \\
\hline & & \multirow{4}{*}{$\begin{array}{l}\text { 18. Medidas } \\
\text { ambientales }\end{array}$} & Acceso de la luz solar \\
\hline & & & $\begin{array}{l}\text { Acceso de luz artificial de lámparas de luz } \\
\text { ultravioleta. }\end{array}$ \\
\hline & & & $\begin{array}{l}\text { Ventilación natural proveniente de puertas y } \\
\text { ventanas. }\end{array}$ \\
\hline & & & $\begin{array}{l}\text { Sistemas de ventilación mecánica conductos } \\
\text { de aire, rejillas, filtradores de aire, } \\
\text { ventiladores. }\end{array}$ \\
\hline & & \multirow[t]{4}{*}{$\begin{array}{l}\text { 19. Protección } \\
\text { personal }\end{array}$} & $\begin{array}{l}\text { Hay mascarillas } n .^{\circ} 95 \text { para la atención de } \\
\text { pacientes con tuberculosis. }\end{array}$ \\
\hline & & & $\begin{array}{l}\text { Los pacientes con tos y expectoración usan } \\
\text { tapabocas. }\end{array}$ \\
\hline & & & $\begin{array}{l}\text { Se imparten capacitaciones de cómo usar la } \\
\text { mascarilla n- } 95 \text {. }\end{array}$ \\
\hline & & & $\begin{array}{l}\text { Usted cambia la mascarilla n. }{ }^{\circ} 95 \text { luego de } 6 \\
\text { horas de uso continuo. }\end{array}$ \\
\hline
\end{tabular}

\section{Diseño del instrumento y de escala de valoración}

Posterior a la revisión conceptual de las diferentes posturas y del constructo de vulnerabilidad, el cual incluyó el concepto de condiciones de vida y trabajo, se realizó la conformación de las categorías de abordaje en: 4 dimensiones (Individual/Extralaboral, Condiciones de salud, Condiciones de trabajo, Control de la Infección), así mismo se revisaron 19 subdimensiones, y se efectuó la formulación de los 70 ítems iniciales, los cuales fueron sometidos a revisiones a profundidad, en términos de revisión del lenguaje, redacción, y comprensión.

La composición de los ítems del instrumento IVTS TB- 001, tomó cada uno de sus ítems como unidades básicas de información, teniendo en cuenta su redacción, y claridad, así como también que dieran respuesta a la dimensión y al constructo objeto de estudio. Se evitó la utilización de preguntas en forma negativa, el uso de interrogaciones, y de preguntas con difícil respuesta, o que implicaran la inducción de las respuestas en los trabajadores.

Se realizó el diseño de 70 ítems iniciales, los cuales fueron depurados por los investigadores, para obtener un total de 50 ítems los cuales fueron seleccionados para continuar con el proceso de validación a nivel de validez aparente y de contenido.

Seguidamente se ejecutó la orientación y el orden de los ítems del instrumento teniendo en cuenta su grado de formulación e indagación de las dimensiones y subdimensiones y se realizó la definición de escala tipo Likert. ${ }^{17}$ 


\section{Evaluación de la comprensión en la población objeto IVTS TB- 001}

En relación a la evaluación de la comprensión en el proceso de validez aparente, esta se definió como el entendimiento de lo que se quería decir al leer el ítem, en la población objeto de estudio; en este caso, fue efectuada valoración en un total de $\mathrm{N}^{\circ}=502$ trabajadores de la salud de dos instituciones donde se realizaba atención de personas con diagnóstico de Tuberculosis.

Los trabajadores fueron seleccionados mediante muestreo aleatorio simple, y se realizó la valoración y comprensión de los 50 ítems en cada una de las dimensiones implicadas mediante los siguientes criterios:

1= No se comprende el ítem: el ítem sufrió restructuración o remplazo si el porcentaje de sujetos afirman no comprender el ítem, igual o inferior al $90 \%$.

$\mathbf{2}=$ Se comprende parcialmente El ítem fue objeto de revisión y cambios en verbos, signos de puntuación si el $90 \%$ al $95 \%$ de los sujetos reporta, que comprende parcialmente.

3= Se comprende totalmente: El ítem conservó su redacción, lenguaje y formulación en más del $95 \%$ de trabajadores que comprendieran totalmente el ítem.

A continuación se muestran los resultados de comprensión por dimensiones:

\section{Valoración de la comprensión Ítems Condiciones Individuales/Extralaborales}

Para evaluar la comprensión de los ítems de la dimensión de condiciones de trabajo individuales/extralaborales se realizó aplicación del ítem 1 al 13 en la población obteniendo los siguientes resultados:

Gráfico 1. Comprensión de los ítems 1-13 Dimensión Individual/Extralaboral ITV TB 001

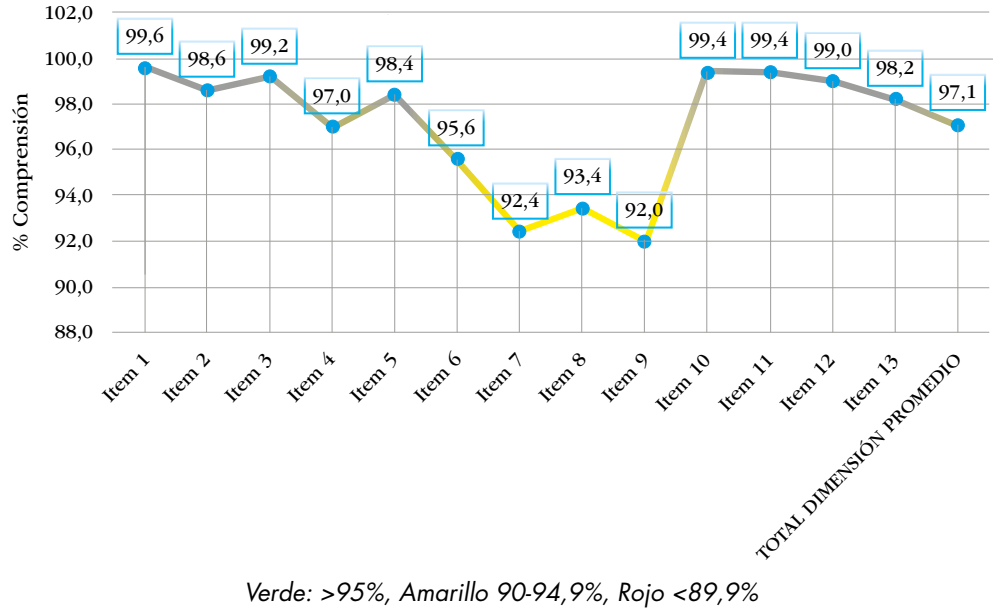

En relación a la comprensión de la dimensión individual y extralaboral 10 de 13 ítems obtuvieron comprensión total en la población objeto.

\section{Valoración de la comprensión Ítems Condiciones de Salud}

Para evaluar la comprensión de los ítems de la dimensión de condiciones de trabajo extra laborales se realizó su aplicación del ítem 14 al 23 obteniendo los siguientes resultados: 
Gráfico 2. Comprensibilidad de los ítems 14-23 Dimensión Condiciones de Salud ITV TB-001

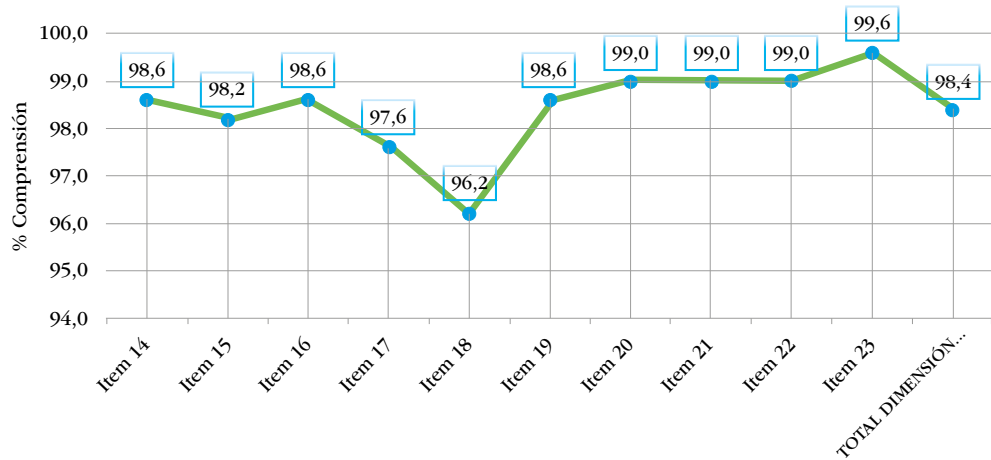

En relación a la comprensión de la dimensión Condiciones de salud 10 de 10 ítems obtuvieron comprensión total en la población objeto.

\section{Valoración de la comprensión Ítems Condiciones de Trabajo}

Para evaluar la comprensión de los ítems de la dimensión de condiciones de trabajo intralaborales se realizó su aplicación del ítem 24 al 37 obteniendo los siguientes resultados;

Gráfico 3. Comprensibilidad de los ítems 24-37 Dimensión Condiciones Intralaborales ITV TB 001

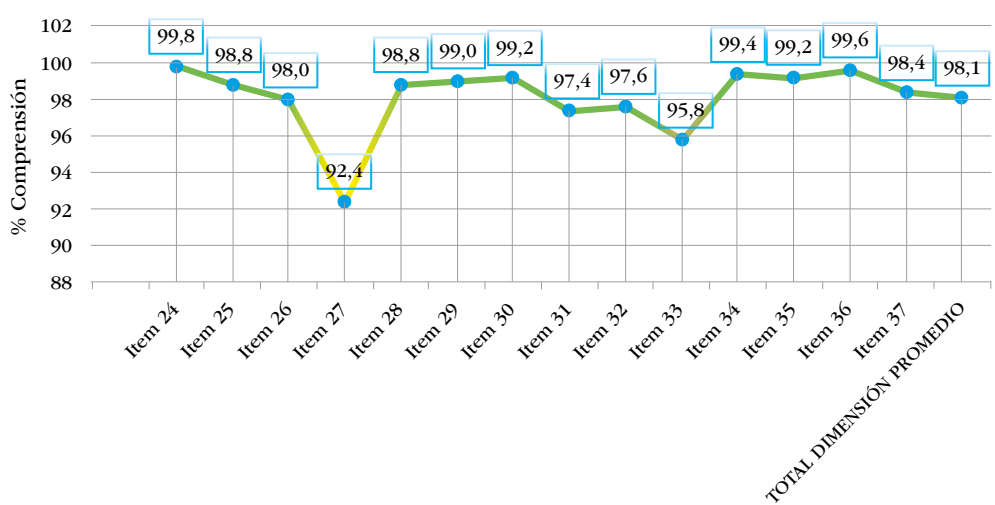

En relación a la comprensión de la dimensión condiciones intralaborales 13 de 14 ítems obtuvieron comprensión total en la población objeto.

\section{Valoración de la comprensión Dimensión Control de Infección}

Para evaluar la comprensión de los ítems de la dimensión de control de infección, se realizó su aplicación del ítem 38 al 50 obteniendo los siguientes resultados;

Gráfico 4. De los ítems 38-50 Dimensión Control de Infecciones ITV TB 001

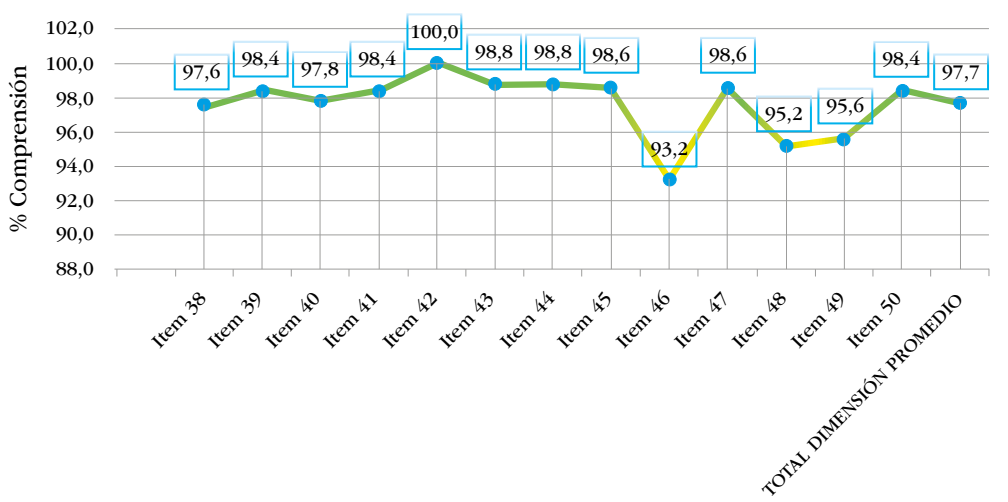


En relación a la comprensión de la dimensión individual y extralaboral 12 de 13 ítems obtuvieron comprensión total en la población objeto.

Frente a los resultados de la valoración de la comprensión en las dimensiones del instrumento IVTS TB 001, se identificó que la mayoría de ítems obtuvieron una comprensión total (>95\%), en la población objeto.

\section{Valoración de claridad del instrumento por expertos}

La "claridad" entendiéndose como la frase o ítem con lenguaje y redacción fácil de comprender, se evaluó mediante un juicio de los 5 expertos, quienes participaron en el proceso de evaluación de la claridad y efectuando consenso promedio mayor o igual a $>0.58$ para ser considerado como ítem claro:

Tabla 3. Valoración de la Claridad de los ítems IVTS TB- 001 por expertos

\begin{tabular}{|c|c|c|c|c|c|c|c|}
\hline \multirow{2}{*}{ ÍTEM } & \multicolumn{7}{|c|}{ CLARIDAD } \\
\hline & $\mathbf{A}$ & $\mathbf{B}$ & C & D & $\mathbf{E}$ & $\mathbf{N} .^{\circ}$ & Índice $=\left(\mathrm{N}^{\circ} \mathrm{e}-(\mathrm{N} / 2)\right) /\left(\mathrm{N}^{\circ} \mathrm{e} / 2\right)$ \\
\hline Ítem 1 & 1 & 1 & 1 & 1 & 1 & 5 & $\mathbf{1}$ \\
\hline Ítem 2 & 1 & 1 & 1 & 1 & 0 & 4 & $\mathbf{0 , 8}$ \\
\hline Ítem 3 & 1 & 1 & 1 & 1 & 1 & 5 & 1 \\
\hline Ítem 4 & 1 & 1 & 1 & 1 & 1 & 5 & $\mathbf{1}$ \\
\hline Ítem 5 & 1 & 1 & 1 & 1 & 1 & 5 & 1 \\
\hline Ítem 6 & 1 & 1 & 1 & 1 & 0 & 4 & 0,8 \\
\hline Ítem 7 & 1 & 1 & 1 & 1 & 1 & 5 & 1 \\
\hline Ítem 8 & 1 & 1 & 0 & 1 & 1 & 4 & 8 \\
\hline Ítem 9 & 0 & 1 & 1 & 1 & 1 & 4 & 8 \\
\hline Ítem 10 & 1 & 1 & 1 & 1 & 1 & 5 & 1 \\
\hline Ítem 11 & 1 & 1 & 1 & 1 & 1 & 5 & 1 \\
\hline Ítem 12 & 1 & 1 & 1 & 1 & 1 & 5 & 1 \\
\hline Ítem 13 & 1 & 1 & 1 & 1 & 1 & 5 & 1 \\
\hline Ítem 14 & 1 & 1 & 0 & 1 & 1 & 4 & 8 \\
\hline Ítem 15 & 1 & 1 & 1 & 1 & 1 & 5 & 1 \\
\hline Ítem 16 & 1 & 1 & 1 & 1 & 1 & 5 & $\mathbf{1}$ \\
\hline Ítem 17 & 1 & 1 & 1 & 1 & 1 & 5 & 1 \\
\hline Ítem 18 & 1 & 1 & 1 & 1 & 0 & 4 & 0,8 \\
\hline Ítem 19 & 1 & 1 & 1 & 1 & 1 & 5 & 1 \\
\hline Ítem 20 & 1 & 1 & 1 & 1 & 1 & 5 & 1 \\
\hline Ítem 21 & 1 & 1 & 1 & 1 & 1 & 5 & 1 \\
\hline Ítem 22 & 1 & 1 & 1 & 1 & 1 & 5 & 1 \\
\hline Ítem 23 & 1 & 1 & 1 & 1 & 1 & 5 & 1 \\
\hline Ítem 24 & 1 & 1 & 1 & 1 & 1 & 5 & 1 \\
\hline Ítem 25 & 1 & 1 & 1 & 1 & 0 & 4 & 0,8 \\
\hline Ítem 26 & 1 & 1 & 1 & 1 & 0 & 4 & 0,8 \\
\hline Ítem 27 & 1 & 1 & 1 & 1 & 0 & 4 & 0,8 \\
\hline Ítem 28 & 1 & 1 & 1 & 1 & 1 & 5 & 1 \\
\hline Ítem 29 & 1 & 1 & 1 & 1 & 1 & 5 & 1 \\
\hline Ítem 30 & 0 & 1 & 1 & 1 & 1 & 4 & 0,8 \\
\hline Ítem 31 & 1 & 1 & 1 & 1 & 0 & 4 & 0,8 \\
\hline
\end{tabular}




\begin{tabular}{|c|c|c|c|c|c|c|c|}
\hline \multirow{2}{*}{ ÍTEM } & \multicolumn{7}{|c|}{ CLARIDAD } \\
\hline & $\mathbf{A}$ & B & C & $\mathbf{D}$ & $\mathbf{E}$ & $\mathbf{N} .^{\circ}$ & Índice $=\left(\mathrm{N}^{\circ} \mathrm{e}-(\mathrm{N} / \mathbf{2})\right) /\left(\mathrm{N}^{\circ} \mathrm{e} / \mathbf{2}\right)$ \\
\hline Ítem 32 & 1 & 1 & 1 & 1 & 1 & 5 & $\mathbf{1}$ \\
\hline Ítem 33 & 1 & 1 & 1 & 1 & 1 & 5 & 1 \\
\hline Ítem 34 & 1 & 1 & 1 & 1 & 0 & 4 & 0,8 \\
\hline Ítem 35 & 1 & 1 & 1 & 1 & 1 & 5 & 1 \\
\hline Ítem 36 & 1 & 1 & 1 & 1 & 1 & 5 & 1 \\
\hline Ítem 37 & 1 & 1 & 1 & 1 & 1 & 5 & 1 \\
\hline Ítem 38 & 1 & 1 & 0 & 1 & 1 & 4 & 0,8 \\
\hline Ítem 39 & 1 & 1 & 1 & 1 & 1 & 5 & 1 \\
\hline Ítem 40 & 0 & 1 & 0 & 1 & 1 & 3 & 0,6 \\
\hline Ítem 41 & 1 & 1 & 1 & 1 & 1 & 5 & 1 \\
\hline Ítem 42 & 1 & 1 & 1 & 1 & 1 & 5 & 1 \\
\hline Ítem 43 & 0 & 1 & 1 & 1 & 1 & 4 & 0,8 \\
\hline Ítem 44 & 1 & 1 & 1 & 1 & 1 & 5 & 1 \\
\hline Ítem 45 & 1 & 1 & 1 & 1 & 1 & 5 & 1 \\
\hline Ítem 46 & 0 & 1 & 1 & 1 & 1 & 4 & 0,8 \\
\hline Ítem 47 & 1 & 1 & 1 & 1 & 1 & 5 & 1 \\
\hline Ítem 48 & 1 & 1 & 1 & 1 & 1 & 5 & 1 \\
\hline Ítem 49 & 1 & 1 & 0 & 1 & 1 & 4 & 0,8 \\
\hline Ítem 50 & 1 & 1 & 1 & 1 & 1 & 5 & 1 \\
\hline & de c & $1 \mathrm{de}$ & $\mathrm{ms}$ & trur & & 4,64 & 0,92 \\
\hline
\end{tabular}

En relación al proceso de valoración de la claridad del instrumento en el juicio de expertos se identificó que los 50 ítems obtuvieron una claridad mayor al $>0.58$ con un índice general de $\mathrm{N}=0,92$, con el cual se cumple el criterio de claridad en todos y cada uno de los ítems.

\section{Valoración de la Precisión del Instrumento por expertos}

La valoración mediante del juicio de expertos determino la precisión de los ítems es decir la formulación puntual, exacta que no diera lugar a ambigüedad o duda en los ítems, esta se consideró aceptable si existía un consenso mayor a $>0.58$ :

Tabla 4. Valoración de la Precisión general de los ítems IVTS TB- 001 por parte de expertos

\begin{tabular}{|c|c|c|c|c|c|c|c|}
\hline \multirow{2}{*}{ ÍTEM } & \multicolumn{7}{|c|}{ PRECISIÓN } \\
\hline & $\mathbf{A}$ & B & C & D & $\mathbf{E}$ & N. ${ }^{\circ}$ & Índice $=\left(\mathrm{N}^{\circ} \mathrm{e}-(\mathrm{N} / \mathbf{2})\right) /\left(\mathrm{N}^{\circ} \mathrm{e} / 2\right)$ \\
\hline Ítem 1 & 1 & 1 & 1 & 1 & 1 & 5 & 1 \\
\hline Ítem 2 & 0 & 1 & 1 & 1 & 1 & 4 & 0,8 \\
\hline Ítem 3 & 1 & 1 & 1 & 1 & 1 & 5 & $\mathbf{1}$ \\
\hline Ítem 4 & 1 & 1 & 1 & 1 & 1 & 5 & 1 \\
\hline Ítem 5 & 1 & 1 & 1 & 1 & 1 & 5 & 1 \\
\hline Ítem 6 & 0 & 1 & 1 & 1 & 1 & 4 & 0,8 \\
\hline Ítem 7 & 1 & 1 & 1 & 1 & 1 & 5 & 1 \\
\hline Ítem 8 & 1 & 0 & 1 & 1 & 1 & 4 & 0,8 \\
\hline
\end{tabular}




\begin{tabular}{|c|c|c|c|c|c|c|c|}
\hline \multirow{2}{*}{ ÍTEM } & \multicolumn{7}{|c|}{ PRECISIÓN } \\
\hline & $\mathbf{A}$ & B & C & $\mathbf{D}$ & $\mathbf{E}$ & $\mathbf{N} .^{\circ}$ & Índice $=\left(\mathrm{N}^{\circ} \mathrm{e}-(\mathrm{N} / 2)\right) /\left(\mathrm{N}^{\circ} \mathrm{e} / \mathbf{2}\right)$ \\
\hline Ítem 9 & 1 & 0 & 0 & 1 & 0 & 2 & 0,4 \\
\hline Ítem 10 & 1 & 1 & 1 & 1 & 0 & 4 & 0,8 \\
\hline Ítem 11 & 1 & 1 & 1 & 1 & 0 & 4 & 0,8 \\
\hline Ítem 12 & 1 & 1 & 1 & 1 & 0 & 4 & 0,8 \\
\hline Ítem 13 & 1 & 0 & 1 & 1 & 1 & 4 & 0,8 \\
\hline Ítem 14 & 1 & 1 & 1 & 1 & 0 & 4 & 0,8 \\
\hline Ítem 15 & 1 & 1 & 1 & 1 & 1 & 5 & 1 \\
\hline Ítem 16 & 1 & 1 & 1 & 1 & 1 & 5 & 1 \\
\hline Ítem 17 & 1 & 1 & 1 & 1 & 1 & 5 & 1 \\
\hline Ítem 18 & 1 & 1 & 1 & 1 & 1 & 5 & 1 \\
\hline Ítem 19 & 1 & 1 & 1 & 1 & 1 & 5 & 1 \\
\hline Ítem 20 & 1 & 1 & 1 & 1 & 1 & 5 & 1 \\
\hline Ítem 21 & 1 & 1 & 1 & 1 & 1 & 5 & 1 \\
\hline Ítem 22 & 1 & 1 & 1 & 1 & 1 & 5 & 1 \\
\hline Ítem 23 & 1 & 1 & 1 & 1 & 0 & 4 & 0,8 \\
\hline Ítem 24 & 1 & 0 & 1 & 1 & 1 & 4 & 0,8 \\
\hline Ítem 25 & 0 & 1 & 1 & 1 & 1 & 4 & 80 \\
\hline Ítem 26 & 0 & 1 & 1 & 1 & 1 & 4 & 0,8 \\
\hline Ítem 27 & 0 & 1 & 1 & 1 & 1 & 4 & 0,8 \\
\hline Ítem 28 & 1 & 1 & 1 & 1 & 1 & 5 & 1 \\
\hline Ítem 29 & 1 & 1 & 1 & 1 & 1 & 5 & 1 \\
\hline Ítem 30 & 1 & 1 & 1 & 1 & 1 & 5 & 1 \\
\hline Ítem 31 & 0 & 1 & 1 & 1 & 1 & 4 & 0,8 \\
\hline Ítem 32 & 1 & 1 & 1 & 1 & 1 & 5 & 1 \\
\hline Ítem 33 & 1 & 1 & 1 & 1 & 1 & 5 & 1 \\
\hline Ítem 34 & 1 & 1 & 1 & 1 & 1 & 5 & 1 \\
\hline Ítem 35 & 1 & 1 & 1 & 1 & 1 & 5 & 1 \\
\hline Ítem 36 & 0 & 1 & 1 & 1 & 1 & 4 & 0,8 \\
\hline Ítem 37 & 1 & 1 & 1 & 1 & 1 & 5 & 1 \\
\hline Ítem 38 & 1 & 1 & 1 & 1 & 0 & 4 & 0,8 \\
\hline Ítem 39 & 1 & 1 & 1 & 1 & 1 & 5 & 1 \\
\hline Ítem 40 & 1 & 1 & 1 & 1 & 0 & 4 & 0,8 \\
\hline Ítem 41 & 1 & 1 & 1 & 1 & 1 & 5 & 1 \\
\hline Ítem 42 & 1 & 1 & 1 & 1 & 1 & 5 & 1 \\
\hline Ítem 43 & 1 & 1 & 1 & 1 & 1 & 5 & 1 \\
\hline Ítem 44 & 1 & 1 & 1 & 1 & 1 & 5 & 1 \\
\hline Ítem 45 & 0 & 1 & 1 & 1 & 1 & 4 & 0,8 \\
\hline Ítem 46 & 0 & 1 & 1 & 1 & 1 & 4 & 0,8 \\
\hline Ítem 47 & 1 & 1 & 1 & 1 & 1 & 5 & 1 \\
\hline Ítem 48 & 1 & 1 & 1 & 1 & 1 & 5 & 1 \\
\hline Ítem 49 & 0 & 1 & 1 & 1 & 0 & 3 & 0,6 \\
\hline Ítem 50 & 1 & 1 & 1 & 1 & 1 & 5 & 1 \\
\hline \multicolumn{6}{|c|}{ Índice de precisión de los ítems del instrumento } & 4,52 & 0,94 \\
\hline
\end{tabular}


En relación al proceso de valoración de la precisión del instrumento en el juicio de expertos se identificó que los 50 ítems obtuvieron una precisión mayor al $>0.58$ con un índice general de $\mathrm{N}=0,94$, con el cual se cumple el criterio de precisión en todos y cada uno de los ítems.

\section{Valoración de la Comprensión del Instrumento por expertos}

La valoración de la comprensión consistió en el entendimiento de lo que se quería decir al leer el ítem. Esta propiedad se valoró sometiendo a juicio de expertos los ítems del instrumento, obteniendo los siguientes resultados:

Tabla 5. Valoración de la Comprensión de los ítems IVTS TB- 001 por parte de expertos

\begin{tabular}{|c|c|c|c|c|c|c|c|}
\hline \multirow{2}{*}{ ÍTEM } & \multicolumn{7}{|c|}{ COMPRENSIÓN } \\
\hline & $\mathbf{A}$ & B & C & $\mathbf{D}$ & $\mathbf{E}$ & $\mathbf{N} .^{\circ}$ & Índice $=\left(\mathrm{N}^{\circ} \mathrm{e}-(\mathrm{N} / 2)\right) /\left(\mathrm{N}^{\circ} \mathrm{e} / \mathbf{2}\right)$ \\
\hline Ítem 1 & 1 & 1 & 1 & 1 & 1 & 5 & 1 \\
\hline Ítem 2 & 0 & 1 & 1 & 1 & 1 & 4 & 0,8 \\
\hline Ítem 3 & 1 & 1 & 1 & 1 & 1 & 5 & 1 \\
\hline Ítem 4 & 1 & 1 & 1 & 1 & 1 & 5 & $\mathbf{0 , 1}$ \\
\hline Ítem 5 & 1 & 1 & 1 & 1 & 1 & 5 & 1 \\
\hline Ítem 6 & 0 & 1 & 1 & 1 & 1 & 4 & 0,8 \\
\hline Ítem 7 & 1 & 1 & 1 & 1 & 1 & 5 & 1 \\
\hline Ítem 8 & 1 & 0 & 0 & 1 & 1 & 3 & 0,6 \\
\hline Ítem 9 & 1 & 0 & 1 & 1 & 1 & 4 & 0,8 \\
\hline Ítem 10 & 1 & 1 & 1 & 1 & 1 & 5 & 1 \\
\hline Ítem 11 & 1 & 1 & 1 & 1 & 1 & 5 & 1 \\
\hline Ítem 12 & 1 & 1 & 1 & 1 & 1 & 5 & 1 \\
\hline Ítem 13 & 1 & 0 & 1 & 1 & 1 & 4 & 0,8 \\
\hline Ítem 14 & 1 & 1 & 0 & 1 & 1 & 4 & 0,8 \\
\hline Ítem 15 & 1 & 1 & 1 & 1 & 1 & 5 & 1 \\
\hline Ítem 16 & 1 & 1 & 1 & 1 & 1 & 5 & 1 \\
\hline Ítem 17 & 1 & 1 & 1 & 1 & 1 & 5 & 1 \\
\hline Ítem 18 & 1 & 1 & 1 & 1 & 1 & 5 & 1 \\
\hline Ítem 19 & 1 & 1 & 1 & 1 & 1 & 5 & 1 \\
\hline Ítem 20 & 1 & 1 & 1 & 1 & 1 & 5 & 1 \\
\hline Ítem 21 & 1 & 1 & 1 & 1 & 1 & 5 & 1 \\
\hline Ítem 22 & 1 & 1 & 1 & 1 & 1 & 5 & 1 \\
\hline Ítem 23 & 1 & 1 & 1 & 1 & 1 & 5 & 1 \\
\hline Ítem 24 & 1 & 0 & 1 & 1 & 1 & 4 & 0,8 \\
\hline Ítem 25 & 0 & 1 & 1 & 1 & 1 & 4 & 0,8 \\
\hline Ítem 26 & 0 & 1 & 1 & 1 & 1 & 4 & 0,8 \\
\hline Ítem 27 & 0 & 1 & 1 & 1 & 1 & 4 & 0,8 \\
\hline Ítem 28 & 1 & 1 & 1 & 1 & 1 & 5 & 1 \\
\hline Ítem 29 & 1 & 1 & 1 & 1 & 1 & 5 & 1 \\
\hline Ítem 30 & 1 & 1 & 1 & 0 & 1 & 4 & 0,8 \\
\hline Ítem 31 & 0 & 1 & 1 & 1 & 1 & 4 & 0,8 \\
\hline Ítem 32 & 1 & 1 & 1 & 0 & 1 & 4 & 0,8 \\
\hline Ítem 33 & 1 & 1 & 1 & 1 & 1 & 5 & 1 \\
\hline
\end{tabular}




\begin{tabular}{|c|c|c|c|c|c|c|c|}
\hline \multirow{2}{*}{ ÍTEM } & \multicolumn{7}{|c|}{ COMPRENSIÓN } \\
\hline & $\mathbf{A}$ & $\mathbf{B}$ & C & D & $\mathbf{E}$ & N..$^{\circ}$ & Índice $=\left(\mathrm{N}^{\circ} \mathrm{e}-(\mathrm{N} / \mathbf{2})\right) /\left(\mathrm{N}^{\circ} \mathrm{e} / \mathbf{2}\right)$ \\
\hline Ítem 34 & 0 & 1 & 1 & 1 & 1 & 4 & $\mathbf{0 , 8}$ \\
\hline Ítem 35 & 0 & 1 & 1 & 1 & 1 & 4 & 0,8 \\
\hline Ítem 36 & 0 & 1 & 1 & 1 & 1 & 4 & 0,8 \\
\hline Ítem 37 & 1 & 1 & 1 & 1 & 1 & 5 & 1 \\
\hline Ítem 38 & 1 & 1 & 0 & 1 & 1 & 4 & 0,8 \\
\hline Ítem 39 & 1 & 1 & 1 & 1 & 1 & 5 & $\mathbf{1}$ \\
\hline Ítem 40 & 1 & 1 & 0 & 1 & 1 & 4 & 0,8 \\
\hline Ítem 41 & 1 & 1 & 1 & 1 & 1 & 5 & 1 \\
\hline Ítem 42 & 0 & 1 & 1 & 1 & 1 & 4 & 0,8 \\
\hline Ítem 43 & 0 & 1 & 1 & 1 & 1 & 4 & 0,8 \\
\hline Ítem 44 & 1 & 1 & 1 & 1 & 1 & 5 & 1 \\
\hline Ítem 45 & 0 & 1 & 1 & 1 & 1 & 4 & 0,8 \\
\hline Ítem 46 & 0 & 1 & 1 & 1 & 1 & 4 & 0,8 \\
\hline Ítem 47 & 1 & 1 & 1 & 1 & 1 & 5 & 1 \\
\hline Ítem 48 & 1 & 1 & 1 & 1 & 1 & 5 & 1 \\
\hline Ítem 49 & 0 & 1 & 0 & 1 & 1 & 3 & 0,8 \\
\hline Ítem 50 & 1 & 1 & 1 & 1 & 1 & 5 & 1 \\
\hline \multicolumn{6}{|c|}{ Índice de comprensión de los ítems del instrumento } & 4,5 & 0,90 \\
\hline
\end{tabular}

En relación al proceso de valoración de la comprensión del instrumento en el juicio de expertos se identificó que los 50 ítems obtuvieron una comprensión mayor al $>0.58$ con un índice general de comprensión de $\mathrm{N}=0.90$, con lo cual se cumplió el criterio de comprensión en todos y cada uno de los ítems.

\section{Valoración de la validez de contenido (Relevancia)}

Teniendo en cuenta la fase de validación de contenido se efectuó el desarrollo de un juicio de expertos para observar la relevancia de los 50 ítems del instrumento IVTS TB -001, clasificando cada ítem como:

- Relevante.

- Poco Relevante.

- No relevante.

Para tal efecto se realizó aplicación del Índice de Validez de Contenido y la Razón de validación de contenido definidos por Lawshe, 1977 y su ajuste en el modelo modificado por Tristán, 2007. ${ }^{18}$

Con la aplicación de este modelo se logró tener un Índice de Validación de Contenido o CVR', en la cual se estableció la constante mínima de validación mayor o igual a $>0.5823$ para cualquier número de expertos. A continuación se muestran los resultados de la aplicación de ese modelo: 
Tabla 6. Razón de Validez de los ítems IVTS TB- 001 por parte de expertos aplicando modelo de Lawshe modificado

\begin{tabular}{|c|c|c|c|}
\hline ÍTEM & $\mathbf{N} .^{\circ}$ expertos & N. ${ }^{\circ}$ Consensos Relevante & $\begin{array}{c}\text { CVR': }^{\prime} \text { Modelo Modificado } \\
\text { Lawshe'0,58 } \\
\text { N/n }\end{array}$ \\
\hline Ítem 1 & 5 & 5 & 1,00 \\
\hline Ítem 2 & 5 & 3 & 0,60 \\
\hline Ítem 3 & 5 & 5 & 1,00 \\
\hline Ítem 4 & 5 & 3 & 0,60 \\
\hline Ítem 5 & 5 & 3 & 0,60 \\
\hline Ítem 6 & 5 & 4 & $\mathbf{0 , 8 0}$ \\
\hline Ítem 7 & 5 & 5 & 1,00 \\
\hline Ítem 8 & 5 & 4 & $\mathbf{0 , 8 0}$ \\
\hline Ítem 9 & 5 & 4 & $\mathbf{0 , 8 0}$ \\
\hline Ítem 10 & 5 & 5 & 1,00 \\
\hline Ítem 11 & 5 & 4 & $\mathbf{0 , 8 0}$ \\
\hline Ítem 12 & 5 & 5 & $\mathbf{1 , 0 0}$ \\
\hline Ítem 13 & 5 & 4 & $\mathbf{0 , 8 0}$ \\
\hline Ítem 14 & 5 & 5 & 1,00 \\
\hline Ítem 15 & 5 & 4 & $\mathbf{0 , 8 0}$ \\
\hline Ítem 16 & 5 & 4 & $\mathbf{0 , 8 0}$ \\
\hline Ítem 17 & 5 & 5 & 1,00 \\
\hline Ítem 18 & 5 & 5 & 1,00 \\
\hline Ítem 19 & 5 & 5 & 1,00 \\
\hline Ítem 20 & 5 & 5 & 1,00 \\
\hline Ítem 21 & 5 & 5 & 1,00 \\
\hline Ítem 22 & 5 & 5 & 1,00 \\
\hline Ítem 23 & 5 & 5 & 1,00 \\
\hline Ítem 24 & 5 & 5 & 1,00 \\
\hline Ítem 25 & 5 & 4 & 0,80 \\
\hline Ítem 26 & 5 & 4 & 0,80 \\
\hline Ítem 27 & 5 & 4 & 0,80 \\
\hline Ítem 28 & 5 & 5 & 1,00 \\
\hline Ítem 29 & 5 & 5 & 1,00 \\
\hline Ítem 30 & 5 & 5 & 1,00 \\
\hline Ítem 31 & 5 & 5 & 1,00 \\
\hline Ítem 32 & 5 & 5 & 1,00 \\
\hline Ítem 33 & 5 & 5 & 1,00 \\
\hline Ítem 34 & 5 & 4 & 0,80 \\
\hline Ítem 35 & 5 & 3 & 0,60 \\
\hline Ítem 36 & 5 & 4 & 0,80 \\
\hline Ítem 37 & 5 & 5 & 1,00 \\
\hline Ítem 38 & 5 & 5 & 1,00 \\
\hline Ítem 39 & 5 & 5 & 1,00 \\
\hline Ítem 40 & 5 & 5 & 1,00 \\
\hline Ítem 41 & 5 & 5 & 1,00 \\
\hline
\end{tabular}




\begin{tabular}{lccc}
\hline ÍTEM & N. ${ }^{\circ}$ expertos & N. ${ }^{\circ}$ Consensos Relevante & $\begin{array}{c}\text { CVR': Modelo Modificado } \\
\text { Lawshe' 0,58 } \\
\mathbf{N} / \mathbf{n}\end{array}$ \\
\hline Ítem 42 & 5 & 4 & $\mathbf{0 , 8 0}$ \\
Ítem 43 & 5 & 3 & $\mathbf{0 , 6 0}$ \\
Ítem 44 & 5 & 5 & $\mathbf{1 , 0 0}$ \\
Ítem 45 & 5 & 5 & $\mathbf{1 , 0 0}$ \\
Ítem 46 & 5 & 5 & $\mathbf{1 , 0 0}$ \\
Ítem 47 & 5 & 5 & $\mathbf{1 , 0 0}$ \\
Ítem 48 & 5 & 5 & $\mathbf{1 , 0 0}$ \\
Ítem 49 & 5 & 5 & $\mathbf{1 , 0 0}$ \\
Ítem 50 & 5 & 5 & $\mathbf{1 , 0 0}$ \\
\hline & Todos los ítems CVI & $\mathbf{0 , 9 1}$ \\
\hline
\end{tabular}

En relación a la Razón de validez de contenido dada por los expertos con la modificación del modelo de Lawshe, se obtuvo un índice de validación del 100\% de ítems puesto que estos superan la constante mínima de validación de 0,5823. Por tanto se obtiene un índice de validación general y de cada uno de los ítems.

\section{DISCUSIÓN}

Según Alarcón, el diseño de un instrumento de medición es un proceso complejo, en el cual se requiere la formulación y reformulación de la hipótesis, la documentación referencial, así como también, la selección del constructo, de las dimensiones y subdimensiones, sobre las cuales se plantearan los ítems o las unidades básicas de análisis de información en un instrumento. ${ }^{19}$

Para tal efecto, el diseño y validación del instrumento IVTS TB -001, luego del abordaje teórico-conceptual y de la problematización del objeto de estudio, efectúo la formulación de las dimensiones, subdimensiones e ítems, integrando el desarrollo conceptual de condiciones de trabajo y salud con el constructo de vulnerabilidad de los trabajadores de la salud a la tuberculosis: Este fue definido como: «El conjunto de factores que aumentan o limitan la posibilidad de exposición y contagio con tuberculosis en los trabajadores de la salud, derivados de las dimensiones individuales/extralaborales, las condiciones de salud, las condiciones de trabajo y la dimensión de control de la infección tuberculosa presentes en las instituciones de salud o en el contexto de los trabajadores».

Tal como lo plantea Betancourt, tradicionalmente en el campo de la salud ocupacional se han integrado herramientas e instrumentos con una elevada precisión, para evaluar variables relacionadas con el medio ambiente físico del trabajo como la iluminación, el ruido, la temperatura, la humedad, los contaminantes, químicos, los factores ergonómicos. Sin embargo, la indagación de factores en el contexto de la vida extralaborales/individuales, de los trabajadores y aquellos relacionados a la organización del trabajo son fundamentales para la comprensión e intervención de los agentes de riesgo.

En coherencia a lo anterior, Muñoz y Bertolozzi ${ }^{20}$ plantean que en el análisis de la vulnerabilidad de los trabajadores de la salud a la tuberculosis, es necesario involucrar la identificación de los factores individuales/extralaborales en el sentido en que por ejemplo, los conocimientos de los trabajadores, se ven influenciados por los modos de acceso a la información, y capacitación, lo que infiere, en la percepción del riesgo de los trabajadores de la salud frente a los mecanismos de transmisión y protección ante la Tuberculosis en 
sus lugares de trabajo. Desde el proceso de valoración del instrumento IVTS TB-001 se incluyeron varios aspectos relacionados al ambiente físico y de las condiciones de trabajo extralaborales fundamentales en el análisis de la problemática objeto de estudio.

De otro lado, en el proceso de validación del instrumento IVTS TB 001, para obtener la validez aparente, se utilizó la valoración de la comprensión por parte de una muestra considerable de trabajadores de dos instituciones donde se brindaba atención a pacientes con tuberculosis. En este último procedimiento, se valoró la comprensión, la precisión y la claridad de los 50 ítems, obteniendo como resultado, un índice de concordancia simple mayor al > 0.58, obteniendo: (0.90) comprensión, precisión (0.90), y claridad (0.92), lo cual implicó que el instrumento demostró validación aparente de sus ítems tanto en la población objeto de estudio, como en los expertos seleccionados.

Otros estudios en el campo de la salud y seguridad en el trabajo, mencionan el desarrollo de etapas de validación de instrumentos en su mayoría indagan factores de riesgo psicosociales laborales, y de la salud mental, los cuales han aplicado procedimientos de validación en escalas de estrés laboral, el Mobbing laboral, han aplicado la validez aparente, adaptación cultural y de contenido, como pasos fundamentales y claves en los procesos de construcción y diseño de herramientas o instrumentos en el campo de la salud y seguridad en el trabajo. ${ }^{21,22}$

Los 50 ítems del instrumento IVTS TB- 001, fueron calificados como relevantes, por parte de los expertos, obteniendo un Índice de Validación de Contenido o CVR' acorde al modelo planteado por Lawshe ${ }^{23}$ y modificado por Tristán, del $\mathrm{N}=0.91$ de relevancia en sus ítems, es decir el instrumento demostró validez de contenido general y en cada uno de sus ítems. ${ }^{24}$

Tal como lo plantea Carvajal ${ }^{25}$, Argimon $^{26}$ y otros autores, la validez aparente es una forma de validación en la cual se mide el grado en el que los ítems parecen medir lo que se proponen, y que debe incluir la percepción de la población, como el juicio de los expertos. Esta etapa de validación aparente en el instrumento IVTS TB- 001, implicó el acercamiento con la población de estudio, y de los expertos, lo cual posibilitó obtener la valoración de la comprensibilidad de los ítems, en su lenguaje, redacción y precisión de los ítems, de manera concreta y apropiada a fin de efectuar su aplicación útil para la toma de intervenciones.

Ahora bien, al plantear y reconocer la tuberculosis como una problemática no solo ligada a la salud pública sino también a nivel laboral, en el contexto de los trabajadores del sector salud, implica ampliar el enfoque conceptual y metodológico no solo analizado desde las condiciones del ambiente de trabajo, sino integrar en su desarrollo la valoración y perfil del estado de salud de los trabajadores, y las condiciones individuales y extralaborales de manera integrada. En la construcción y validación de instrumentos con propiedades psicométricas se debe trascender de la visión de la salud ocupacional tradicional, hacia la aplicación del modelo conceptual de la salud, seguridad y ambiente de trabajo.

En el aporte del constructo de vulnerabilidad referenciado por Bertolozzi y Terra ${ }^{27}$, se menciona que la relación individual y de los colectivos, integra los factores derivados de las condiciones de salud y vida, los aspectos clínicos y biológicos, la identificación de las desigualdades sociales, lo que puede hacer más vulnerables a unos individuos en relación a otros, al desarrollo de la tuberculosis.

En este sentido para la actual investigación la vulnerabilidad entendida como los factores que aumentan o limitan la posibilidad de exposición y contagio con tuberculosis en los trabajadores de la salud, derivados de las dimensiones individuales/extralaborales, las condiciones de salud, las condiciones de trabajo y control de la infección, implica un análisis integral de los factores que inciden en la vulnerabilidad de los trabajadores en los diferentes niveles desde la atención primaria en salud, hasta la atención de alta complejidad.

Finalmente, se destaca en relación al diseño y validación del instrumento IVTS TB-001, como un aporte significativo a nivel conceptual- operacional en el campo de la 
salud y seguridad en el trabajo, y referente conceptual de la aplicación del modelo de condiciones de trabajo intra y extralaborales, y de salud, integrado al constructo de Vulnerabilidad en salud, que aporte a la ciencia, tecnología e innovación en el campo de estudio. De igual manera, el instrumento se convierte en una herramienta, útil, y práctica, que al ser aplicada en los escenarios de trabajo del sector salud, permita sustentar bases sólidas de intervención para la prevención y control de la tuberculosis en los trabajadores que cuidan la salud.

\section{AGRADECIMIENTOS}

Los autores agradecen a la Universidad Nacional de Colombia, a la Facultad de Enfermería y a la Maestría en Salud y Seguridad en el Trabajo, centro de excelencia académica innovación e investigación.

\section{REFERENCIAS BIBLIOGRÁFICAS}

1. Laurell A. Procesos Laborales y Patrones de Desgaste en los trabajadores. El obrero en México; 1985.

2. Feo O. Reflexiones sobre la globalización y su impacto sobre la salud de los trabajadores y el ambiente. Ciênc. saúde coletiva. [on the Internet]. 2003. [cited 2011 Mar 26].

3. Organización Panamericana de la Salud. Salud y Seguridad de los Trabajadores del Sector salud. Manual para gerentes y administradores. 2005. (3): 185- ISBN 9275325820.

4. Neffa, JC. Las condiciones y el medio ambiente de trabajo (CyMAT). Presentación de la concepción dominante y una visión alternativa. 1995. CYMAT $\mathrm{N}^{\circ}$ 1. PIETTE-CONICE.

5. World Health Organization-WHO. Global Tuberculosis Control: Surveillance, planning, financing. WHO Report. 2014. ISBN 978924156438 0 Disponible en http://www.who.int/tb/publications/global_ report/2011/gtbr11_full.pdf.

6. Organización Internacional del Trabajo -OIT. Enciclopedia de Salud y Seguridad. Prevención Control y vigilancia de la Tuberculosis. [on the Internet]. 1998. 7-81p. "available on»: http://www.facmed.unam.mx/ deptos/salud/censenanza/spivst/spiv/indexoit.htm.

7. Fica C Alberto, Cifuentes D Marcela, Ajenjo H M. Cristina, Jemenao P M. Irene, Zambrano O Alejandra, Febré V Naldy et al. Tuberculosis en el personal de salud. Rev. chil. infectol. [serial on the Internet]. 2008 Aug [cited 2011 July 25]; 25(4): 243-255. Available from: http://www.scielo.cl/scielo.php?script=sci_ arttext\&pid=S0716-10182008000400001\&lng=en. doi: 10.4067/S0716-10182008000400001.

8. Secretaria Distrital de Salud de Bogotá. Datos reportados de casos Programa Distrital de Control de la Tuberculosis. 2014.

9. Nichiata LY, Bertolozzi MR, Takahashi RF, Fracolli LA. A utilização do conceito "Vulnerabilidade» pela enfermagem. Rev. Latino-Am. Enfermagem [On the Internet]. 2008 Oct [cited 2015 Aug 08]; 16( 5 ): 923-928p.

10. Polit H. Investigación científica en ciencias de la salud: Diseños de investigación para estudios cuantitativos. Capítulo 8, Quinta Edición. México: McGraw Hill. 2000; 203.

11. Sánchez R, Echeverry J. Validación de escalas de medición en salud. Rev. salud pública [Internet]. 2004 Nov [cited 2015 Aug 09]; 6( 3 ): 302-318.

12. Martin MC. Diseño y validación de cuestionarios. Instituto de investigación de enfermedades Raras. Instituto de Salud Carlos III Madrid. Matronas Profesión. 2004.

13. Fortin MF. El proceso de investigación: de la concepción a la realización. México: Ed. McGraw-Hill Interamericana, 1999; 182-183.

14. Tristán AL, Molgado DR. Tablas de Validación de contenido. Instituto de Evaluación e Ingeniería Avanzada, S.C. Mariano Jiménez 1830. ${ }^{a}$ Col. Balcones del Valle 78280 San Luis Potosí, México. 21 de Abril, 2007.

15. Rodríguez M, Lopera J. Conceptos básicos de la validación de escalas de salud mental. Rev Ces Med. 2002; 16(3):31-39.

16. Hernández R, Fernández Collado C, Baptista P. Metodología de la Investigación. 2004. 3. ${ }^{a}$ ed. México: McGraw Hill.

17. Ruiz FD, Barón FJ. Bioestadística Universidad de Málaga. Ed Thompson ISBN 84-9732-341-6. España 2005.

18. Lawshe C.H. A quantitative approach to content validity. Personnel Psychology. 28, 563-575. 
19. Alarcón M, Muñoz NS. Medición en salud: Algunas consideraciones metodológicas. Rev. méd. Chile [revista en la Internet]. 2008 Ene [citado 2015 Jul 05]; 136( 1 ): 125-130. Disponible en: http://www.scielo.cl/scielo.php?script=sci_ arttext\&pid=S0034-98872008000100016\&lng=es. http://dx.doi.org/10.4067/S0034-98872008000100016.

20. Muñoz AI, Bertolozzi MR. "Conhecimentos e atitudes de universitarios em relacao a tuberculose - Boletim de Pneumologia Sanitaria ISSN 0103-460X». En: Brasil Revista Brasileira De Epidemiologia ISSN: 1415790X Ed: Fiocruz.

21. Carvallo A. et al. Adaptation and validation of an oral anticoagulation measurement of treatment adherence instrument. Rev. Latino-Am. Enfermagem [online]. 2010, vol.18, n.3, pp. 301-308. ISSN 01041169., Moreno J, Garrosa E. González J. El desgaste profesional de enfermería Desarrollo y validación de un instrumento. Departamento de Psicología Biológica y de Salud, Facultad de Psicología, Universidad Autónoma de Madrid. Revista de Prevención de Riesgos Laborales 3,1 2000 pag, 18-28.

22. Hernández I, Nino PC, González A. et al. Validación de un instrumento p ara el diagnóstico del mobbing. Cuestionario de Heinz Leymannn (LIPT) modificado. Revista Cubana de Salud y Trabajo 2008;9(1):34-48.

23. Lawshe C.H. Op.Cit 18.

24. Tristán, Agustín: Modificación del modelo de Lawshe para el dictamen cuantitativo de la validez de contenido de un instrumento objetivo. En: Avances en Medición. 2008. Vol. 6, p. 37-48.

25. Carvajal C, Centeno R, Watson M, Martínez Á, Sanz R,. ¿Cómo validar un instrumento de medida de la salud?. Salud pública de México / vol. 55, no. 1, enero-febrero de 2013.

26. Argimon JM, Jiménez J. Métodos de investigación clínica y epidemiológica. 3a. ed. Madrid: Elsevier España, 2004.

27. Terra, MF, Bertolozzi, MR. ¿El tratamiento con supervisión directa ( (DOTS») contribuye para la adhesión al tratamiento de la tuberculosis?. Rev. Latino-Am. Enfermagem [online]. 2008, vol.16, n.4 [cited 2013-0609], pp. 659-664. Available from: http://www.scielo.br/scielo.php.

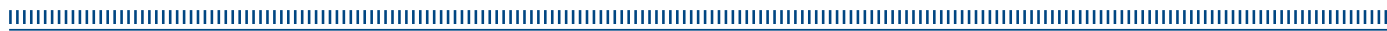

